\title{
De novo transcriptome assembly of drought tolerant CAM plants, Agave deserti and Agave tequilana
}

Stephen M Gross ${ }^{1,2}$, Jeffrey A Martin ${ }^{1,2}$, June Simpson³, María Jazmín Abraham-Juarez ${ }^{3}$, Zhong Wang ${ }^{1,2}$ and Axel Visel ${ }^{1,2^{*}}$

\begin{abstract}
Background: Agaves are succulent monocotyledonous plants native to xeric environments of North America. Because of their adaptations to their environment, including crassulacean acid metabolism (CAM, a water-efficient form of photosynthesis), and existing technologies for ethanol production, agaves have gained attention both as potential lignocellulosic bioenergy feedstocks and models for exploring plant responses to abiotic stress. However, the lack of comprehensive Agave sequence datasets limits the scope of investigations into the molecular-genetic basis of Agave traits.

Results: Here, we present comprehensive, high quality de novo transcriptome assemblies of two Agave species, $A$. tequilana and A. deserti, built from short-read RNA-seq data. Our analyses support completeness and accuracy of the de novo transcriptome assemblies, with each species having a minimum of approximately 35,000 protein-coding genes. Comparison of agave proteomes to those of additional plant species identifies biological functions of gene families displaying sequence divergence in agave species. Additionally, a focus on the transcriptomics of the A. deserti juvenile leaf confirms evolutionary conservation of monocotyledonous leaf physiology and development along the proximal-distal axis.
\end{abstract}

Conclusions: Our work presents a comprehensive transcriptome resource for two Agave species and provides insight into their biology and physiology. These resources are a foundation for further investigation of agave biology and their improvement for bioenergy development.

Keywords: RNA-seq, Bioenergy, Crassulacean acid metabolism, de novo transcriptome assembly JEL Classification codes: Q420 Alternative energy sources

\section{Background}

The lack of genomic and transcriptomic sequence information for agaves, succulent plants native to the arid regions of North America, limits molecular investigation of their adaptations to the abiotic stresses of xeric environments. Agaves are remarkably resistant to heat and drought stress as they employ crassulacean acid metabolism (CAM) - a water-efficient form of photosynthesis in which the uptake of $\mathrm{CO}_{2}$ into plant tissues through stomata and the fixation of $\mathrm{CO}_{2}$ into organic molecules is temporally separated [1]. CAM plants have high water use efficiency, 4-2X more efficient in water use efficiency than plants employing C3

\footnotetext{
* Correspondence: avisel@lbl.gov

'DOE Joint Genome Institute, Walnut Creek, CA, USA

${ }^{2}$ Genomics Division, Lawrence Berkeley National Laboratory, One Cyclotron Road, Berkeley, CA 94720, USA

Full list of author information is available at the end of the article
}

and $\mathrm{C} 4$ photosynthesis [2]. Moreover, an increased $\mathrm{CO}_{2}$ concentration within CAM plant cells increases the efficiency of carbon fixation by Rubisco [2]. Agaves exhibit equally important morphological adaptations to xeric environments that further increase their drought and heat resistance [3]. Specialized leaves [4,5], cuticles [6-8], and roots $[9,10]$ further protect agaves from thermal damage and prolonged drought. Agaves thus offer an opportunity to study broad-spectrum heat and drought resistance not necessarily present in all CAM plants, and provide an important model for creating applied solutions to agricultural challenges associated with climate change $[1,11]$. Because of adaptations to arid environments [5,12], agaves have also recently been proposed as a lignocellulosic bioenergy feedstock suitable for marginal land $[13,14]$.

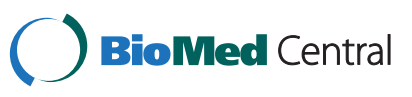


To date, the ecology and physiology of two Agave species, A. tequilana (Figure 1A) and $A$. deserti (Figure 1B), have been studied most extensively. Agave tequilana Weber var. azul, colloquially known as the blue agave, is cultivated in western Mexico for the production of the distilled spirit tequila [15]. A. tequilana is of both cultural $[15,16]$ and economic importance to Mexico, representing $\$ 1.7$ billion in annual revenue within the United States alone [17]. Because of its productivity, established agricultural practices, and ethanol conversion technologies, A. tequilana and its close relatives represent some of the most promising Agave species for bioenergy [18]. Agave deserti, subject of numerous ecological and physiological studies [reviewed in 19], is native to the Sonoran Desert regions of the Southwestern United States and Northwestern Mexico [5] and grows within elevation ranges that experience both hot, dry summers and occasional freezing temperatures in winter [20,21]. Adapted to the conditions of its native habitat, $A$. deserti displays exceptional drought and temperature tolerance. Mature $A$. deserti plants survive up to a year without rainfall [4,22], and, in side-by-side comparisons with 14 other Agave species, $A$. deserti displays the largest range of thermotolerance, surviving a temperature range of $77.5^{\circ} \mathrm{C}\left(-16.1^{\circ} \mathrm{C}\right.$ to $\left.61.4^{\circ} \mathrm{C}\right)$ [23]. While $A$. deserti is comparatively smaller and slowergrowing than $A$. tequilana, it provides a valuable model to study molecular and physiological mechanisms of plant drought and heat resistance [19,24,25].

Agaves have large genomes, estimated to be around 4 Gbp [26] with a significant amount of gene duplication due to paleopolyploidy [27] and a high number of repetitive elements [28], presenting significant challenges for genome assembly. To provide a comprehensive and accurate foundation for molecular studies of agaves, herein we present reference transcriptome datasets of $A$. tequilana and $A$. deserti, assembled from deep RNA-seq data. Cross-species comparisons demonstrate high depth and accuracy of the Agave de novo assemblies. Comparative transcriptome profiling provides insights into the molecular and physiological functions along the proximal-distal axis of the $A$. deserti leaf, and demonstrates broad conservation of leaf development and function across monocotyledonous plants. These reference transcriptomes provide resources for further molecular investigations of the Agave genus to enable their use as models for plant adaptations to abiotic stress, and improve agaves for applied bioenergy technologies.

\section{Results}

Deep sequencing of Agave tissues captures the majority of Agave transcripts

Both $A$. tequilana and $A$. deserti spend the majority of their 5-10 year lifespan as vegetative rosettes (Figure 1A, 1B) before a single flowering event followed by rapid senescence [5]. mRNA was harvested from various Agave tissues (Additional file 1: Table S1, Figure S1), and strandspecific cDNA sequencing libraries of specific insert sizes were prepared for Illumina sequencing (Methods). In total, we sequenced 978 million $A$. tequilana and 615 million $A$. deserti RNA fragments using 150 nucleotide paired-end reads (Additional file 1: Tables S2 and S3). To assess coverage of the agave transcriptomes, we plotted the frequency of observing a new unique 25-mer sequence over an increasing number of randomly sampled reads. In both data sets, the 25-mer discovery frequency decreases as sequencing depth increases, and asymptotically levels off at approximately 0.08 (8\%) (Figure 1C). While complimentary datasets, such as completed genomes, will be required to conclusively determine transcriptome coverage, these observations suggest the sequencing depth was sufficient to sample the majority of sequence diversity in agave tissues. Reads from the two Agave datasets were separately assembled into contigs by the de novo transcriptome assembly pipeline Rnnotator [29]. Resulting contigs were grouped by sequence similarity into genetic loci to account for alternative splicing and reduce redundancy in downstream analyses (Methods, Additional file 1: Table S4).

To eliminate contigs derived from commensal organisms, lab contaminants, and artifacts resulting from incorrect assembly [30,31], contigs of non-plant origin were removed (Methods). Analysis of GC content of contigs from the two agave species and contaminating contigs indicates filtering produces high confidence Agave transcriptomes largely free of contamination (Figure 1D). Resulting Agave transcriptome details are summarized in Table 1 (for additional details, Additional file 1: Tables S4-S6). Assembled contigs are of similar length in both species (Figure 1E). Both agaves encode nearly identical numbers of high-confidence proteins ( 35,000 each, Table 1$)$. Transcripts from non-coding loci tend to be less abundant than transcripts from protein-coding loci (Figure 1F) (Wilcoxon rank sum test $p$-value $<0.05$ ).

\section{Sequence comparisons indicate high accuracy and depth of the A. tequilana de novo assembly}

To examine the accuracy of transcript assembly, we complemented our deep short-read sequencing with smaller-scale long-read single-molecule (Pacific Biosciences) sequencing of $A$. tequilana cDNAs [32] (Methods) (Figure 2A). Error-corrected, high quality subreads (N50 = 450 bp, Additional file 1: Figure S2) (Methods) were aligned to the Rnnotator de novo assembly (Figure 2A). We observed that 4,766 of 4,767 subreads are represented in the short-read based de novo Agave transcriptome assembly. We also compared the A. tequilana Rnnotator assembly to all $82 A$. tequilana nucleotide sequences available from GenBank and observed that 81 (98.8\%) are represented in our dataset (Figure 2A). Comparison of our A. tequilana Rnnotator assembly to a set of 12,972 


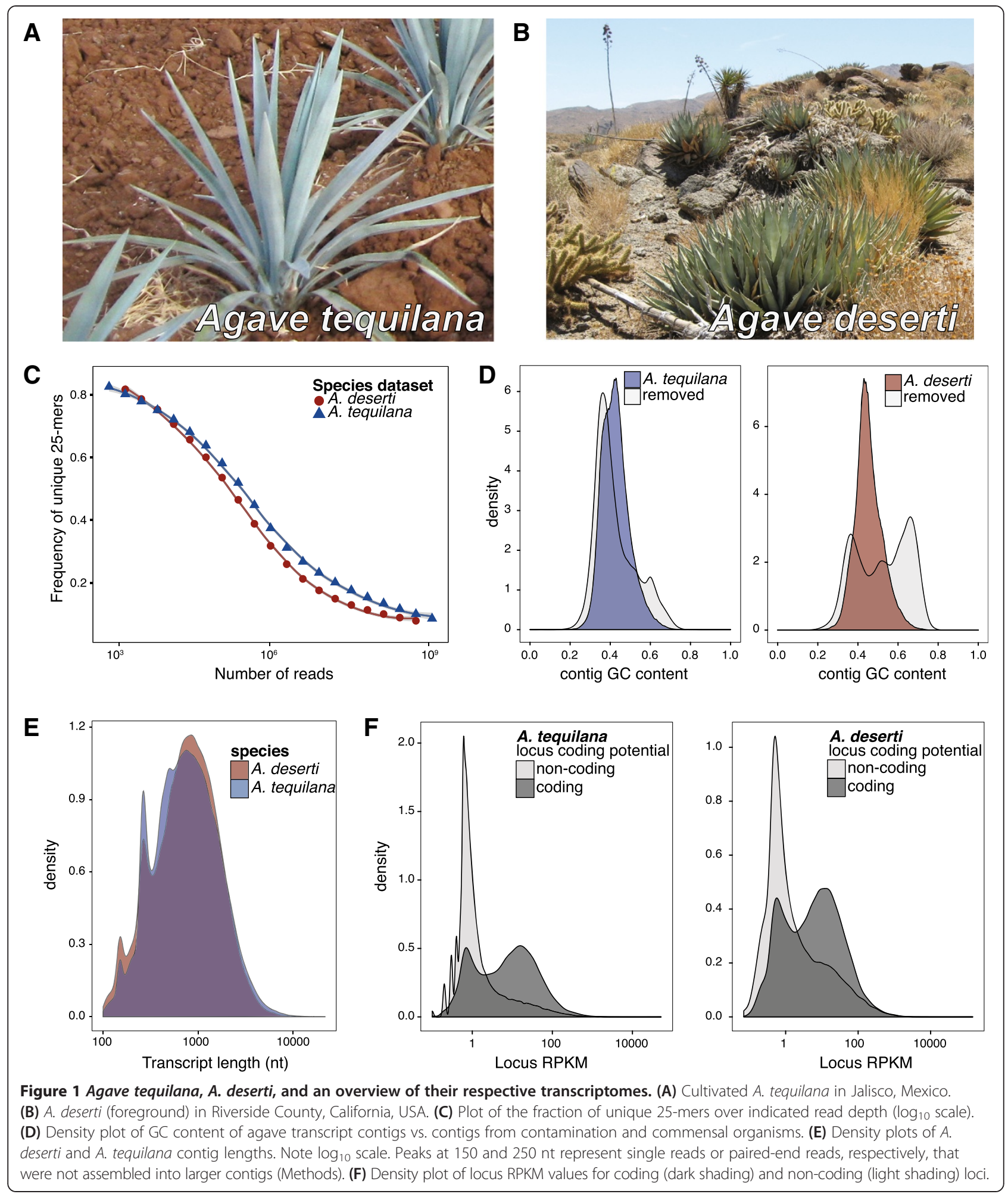

transcripts assembled from low-depth RNA sequencing by McKain et al. [27] (approximately $3 \mathrm{Gbp}$, compared to $293 \mathrm{Gbp}$ in the present study) (Figure 2A) reveals 12,848 of the 12,972 McKain et al. transcripts (99.0\%) are represented in our transcriptome assembly and 8,298 transcript contigs $(64.0 \%)$ align with no insertions or deletions. Of transcript contigs aligning between the two de novo assemblies, 6,578 (50.7\%) are longer in the Rnnotator 
Table 1 Summary of the $A$. tequilana and $A$. deserti transcriptome assemblies

\begin{tabular}{lllllll}
\hline Species & Total sequencing* & No. of Agave loci & $\begin{array}{l}\text { No. Agave } \\
\text { contigs }\end{array}$ & N50 length & $\begin{array}{l}\text { Sum length of } \\
\text { Agave contigs }\end{array}$ & No. of protein-coding loci \\
\hline A. tequilana & $293.5 \mathrm{Gbp}$ & 139,525 & 204,530 & $1387 \mathrm{bp}$ & $204.9 \mathrm{Mbp}$ & 34,870 \\
A. deser & $184.7 \mathrm{Gbp}$ & 88,718 & 128,869 & $1323 \mathrm{bp}$ & $125.0 \mathrm{Mbp}$ & 35,086 \\
\hline
\end{tabular}

Additional details on the depth of Illumina sequencing are in Additional file 1: Tables S2-S6.

*Sequence data from unusable reads where the Illumina TruSeq index could not be deciphered are not included.

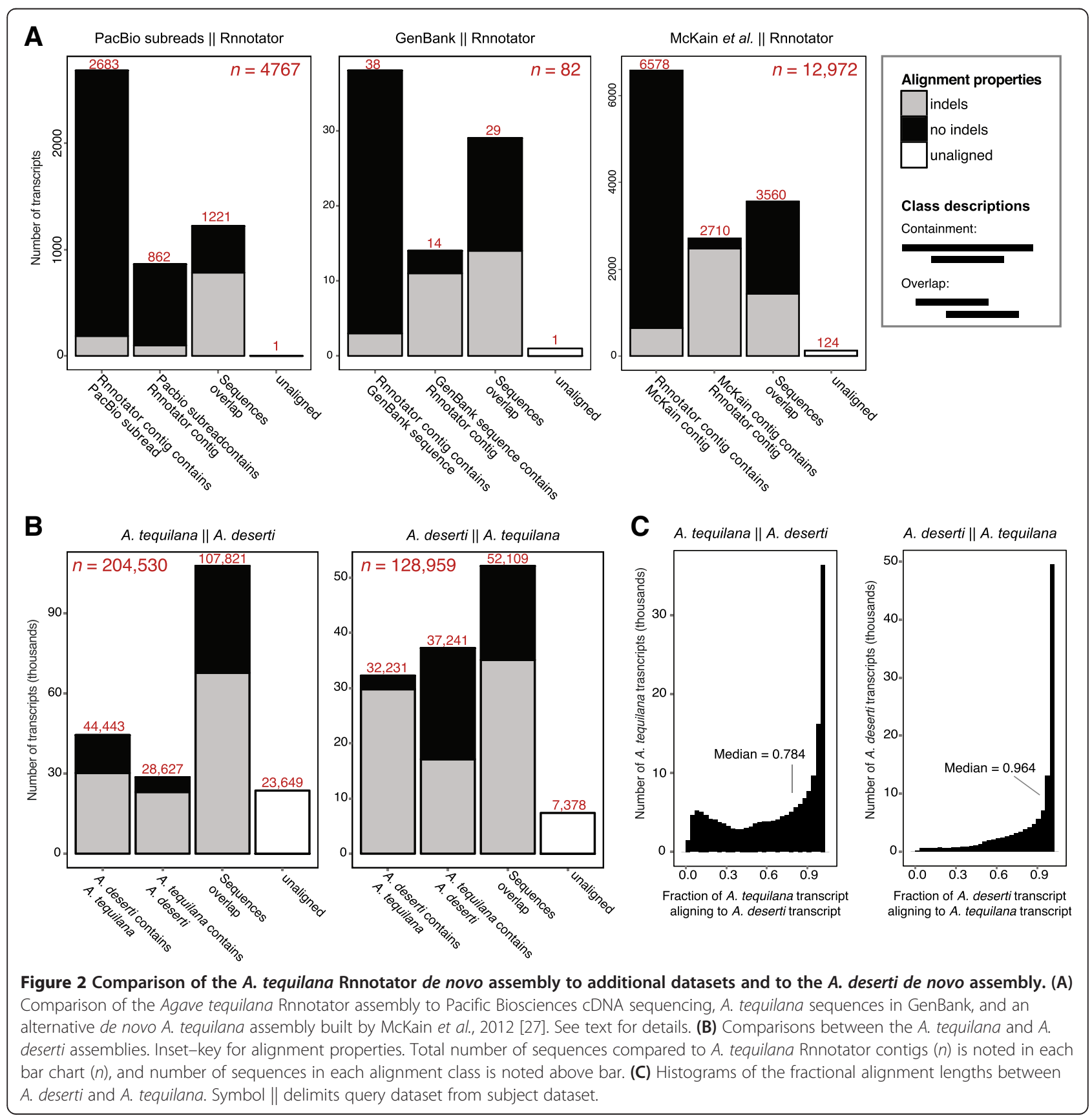


de novo assembly. Taken together, these comparisons further support the accuracy and near-completeness of our reference transcriptome dataset.

\section{Agave deserti and A. tequilana transcriptomes show high sequence identity}

The transcriptomes of $A$. deserti and $A$. tequilana were compared using reciprocal BLAT analyses [33] using a minimum sequence identity threshold of $90 \%$ (Figure $2 \mathrm{~B}$ ). A significant portion of each agave transcriptome aligns to its counterpart, with $94.3 \%$ of $A$. deserti transcripts aligning to A. tequilana, and $88.44 \%$ of $A$. tequilana transcripts aligning to $A$. deserti. Transcripts aligning between the two Agave species also show a significant similarity in length and long regions of sequence alignment (Figure 2C).

\section{Clustering of Agave protein families further support de novo transcriptome completeness}

We identified a core set of 14,709 reciprocal best hit (RBH) protein pairs between the $A$. deserti and $A$. tequilana using BLASTP (Figure 3A). The lengths of these RBH proteins correlate strongly (Pearson $r=0.90$ ) and local alignments demonstrate a median amino acid sequence identity of
98.1\%. This high correspondence between independently assembled datasets further supports assembly accuracy and suggests that a major proportion of the $A$. deserti and $A$. tequilana proteomes are shared between the two species. To further investigate proteomic similarity between agaves, we clustered the Agave proteomes into protein families using OrthoMCL [34]. Most ( 80\%) of the OrthoMCL-defined protein families in $A$. deserti and A. tequilana are common to both species (Figure $3 \mathrm{~B}$ ).

To further substantiate the de novo Agave transcriptomes and perform comparative analyses, Agave proteomes were also clustered by OrthoMCL with the proteomes of 11 additional plant kingdom species obtained from Phytozome [35] (hereafter, Phytozome Tester Set, or PTS, see Methods for details). The PTS includes both monocotyledonous and dicotyledonous plants and plants exhibiting C3 or C4 photosynthesis, but no other CAM plants as no high-quality datasets are currently available in Phytozome. Between $A$. deserti, $A$. tequilana, and the 11 species within the PTS, we obtained 48,133 unique plant protein orthologous groups (hereafter, Plant OGs) from a total of 381,050 proteins [36].

\section{A BLAST}

$1: 1 \mathrm{RBH}$ protein comparison

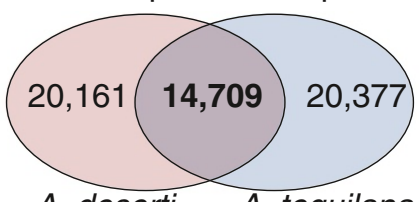

B

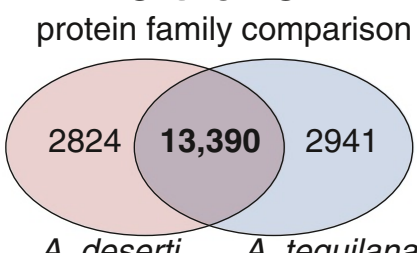

C

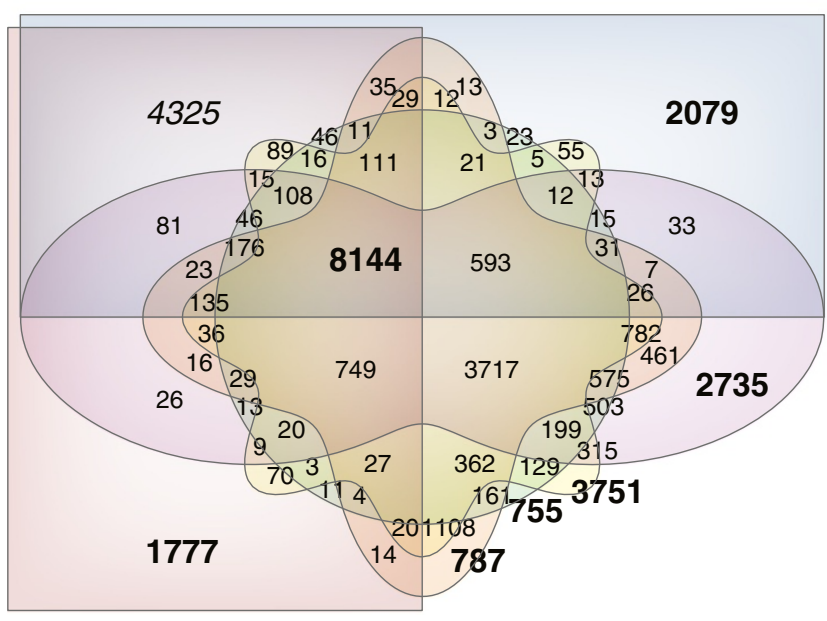

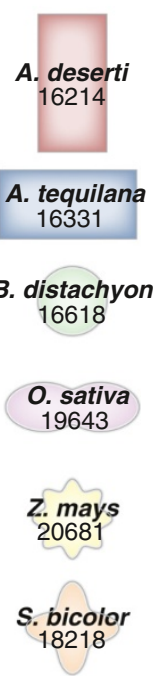

Figure 3 Comparison of inferred proteomes of agaves and additional plant species. (A) Diagram of BLASTP-based 1:1 reciprocal best hit (RBH) proteins shared between agaves. (B) Diagram of OrthoMCL-defined protein families shared between agaves. (C) Diagram of OrthoMCLdefined plant orthologous-group protein families (Plant OGs) shared between agaves and 4 additional monocotyledonous plant species. Shape and color used for each species is noted with the total number of Plant OGs within each species. 
Using OrthoMCL data, we first compared protein lengths between the inferred proteomes of the PTS and our de novo Agave assemblies to address transcript contig completeness. There are 12,346 Plant OGs shared between either $A$. deserti or $A$. tequilana and at least one member of the PTS. These 12,346 Plant OGs encompass 55,676 Agave proteins and 173,611 proteins from the 11 species in the PTS (data available online [36]). The median lengths of Agave and PTS proteins within each of the 12,346 Plant OGs correlate highly (Pearson $r=0.85$ ) and overall demonstrate 1:1 correspondence in protein lengths (best-fit slope $=0.9942$ ) (Additional file 1: Figure S3A). The median length of Agave proteins within the set is $\sim 11 \%$ shorter than that of the PTS (Agave, 356 amino acids; PTS, 389 amino acids; Student's $t$-test $p$-value $<0.05$ ) (Additional file 1: Figure S3B).

To estimate Agave proteome completeness, we compared the inferred $A$. tequilana and $A$. deserti proteomes to those of 4 monocotyledonous grass species in the PTS: Brachypodium distachyon, Oryza sativa, Sorghum bicolor, and Zea mays. An Edwards-Venn diagram of Plant OGs (Figure 3C) demonstrates that 8144 of 13,203 (61.7\%) of protein families common to the 4 grass species are shared with agaves despite approximately 120 million years of evolution separating these grasses (order Poales [37]) and agaves (order Asparagales [37]) [38,39].

The 4325 Plant OGs common to both agaves but absent in four grass species (Figure 3C) represent either Agave protein families not present in grasses or protein families with enough sequence diversity to escape orthology detection by OrthoMCL. Gene ontology (GO) enrichment indicates regulatory diversity separates agaves from other monocots (Additional file 1: Table S7). Abundant transcription factor families within this set include MYB (InterPro IPR014778; 84 Plant OGs), ethylene response factor-domain (AP2/ERF-domain, IPR001471; 48 Plant OGs), C3HC4 Zinc finger (IPR018957; 44 Plant OGs), and WRKY (IPR003657; 41 Plant OGs). This agave-specific set also includes Hsp20-type heat shock proteins (IPR002068; 18 Plant OGs), suggestive of sequence divergence in these agave proteins regulating responses to heat.

\section{Agave protein families are of comparable size to those in other plant species}

Agaves may have adapted to hot, arid environments through expansion of protein families involved in abiotic stress resistance. A comparison of 41,425 OrthoMCLdefined protein families common to any member of the PTS species and either Agave species failed to discover significantly smaller or larger orthologous protein families in agaves (Wilcoxon rank sum test Benjamini-Hochberg corrected $p$-values $>0.05)$. Furthermore, no significant expansion of obvious candidate protein families, such as heat shock proteins (HSPs) [40], heat-shock transcription factors (HSFs) [41], and dehydrins [42] was observed. Thus, using our clustering methodologies, we found no significant expansion of gene families within Agave species suggestive of adaptation to xeric environments. However, the lack of significantly underrepresented PlantOGs supports the completeness of our de novo transcriptome assemblies.

\section{Identifying polymorphisms in $A$. deserti and $A$. tequilana}

Both wild-growing $A$. deserti and traditionally cultivated A. tequilana are expected to harbor significant amounts of heterozygosity. Furthermore, though both A. tequilana and $A$. deserti are cytological diploids [43], recent work indicates agaves are paleopolyploids resulting from two distinct tetraploidization events [27], potentially leading to the presence of highly similar paralagous loci in their genomes. While these issues can potentially complicate the de novo assembly of transcriptomes due to the consolidation of transcripts originating from distinct alleles or paralagous loci into single transcript contigs, these expected polymorphisms provide opportunities to demonstrate the utility of de novo transcriptomes to develop strategies for marker assisted breeding. We attempted to identify loci displaying evidence of combined assembly of polymorphic alleles and/or paralagous genes by mapping reads back to the reference consensus assembly and identifying single-nucleotide polymorphisms (SNPs) or insertions/deletions (indels).

Analysis identified 30,035 (33.9\%) A. deserti loci and 66,701 (47.8\%) A. tequilana loci as having 1 or more highconfidence polymorphism when compared to the reference Illumina de novo assembly (Additional file 1: Figure S4A). The median number of polymorphisms (SNPs or indels) per kilobase (hereafter, PPK) is significantly different between the two species, with $2.066 \mathrm{PPK}$ in $A$. deserti and 4.39 PPK in $A$. tequilana (Wilcoxon rank sum test $p$-value $<0.05)$. Of loci exhibiting polymorphisms, 16,838 (56.1\%) A. deserti and 34732 (52.1\%) A. tequilana loci are protein-coding. In $A$. deserti, non-coding loci exhibit a higher median PPK than coding loci (2.9 vs. 1.6 PPK, respectively, Wilcoxon Rank Sum test $p$-value $<0.05$ ) (Additional file 1: Figure S4), however this was not observed in A. tequilana (4.46 PPK coding, 4.29 PPK non-coding, Wilcoxon Rank Sum test $p$-value $>0.05$ ) (Additional file 1: Figure S4B). Full datasets are available online [36].

\section{Mining Agave proteins for adaptations to xeric environments}

In ex vivo experiments, Agave leaf cells can survive temperatures up to $64.7^{\circ} \mathrm{C}$ [23], suggesting molecular and cellular adaptations contribute to heat tolerance in a manner independent of Agave physiological and morphological adaptations. Though computational prediction of 
protein thermostability from primary structure alone is not completely accurate [44], we tested for protein adaptations to thermal stress using a streamlined version of Thermorank [44] (Additional file 1: Figure S5A, Methods). However, we found no signatures of global, proteome-wide thermotolerance adaptation in agaves (Additional file 1: Figure S5B). Independent tests of Agave proteins within OrthoMCL-defined PlantOGs failed to find agave proteins with significantly higher thermostability than others within Phytozome Tester Set (Wilcoxon rank sum test Benjamini-Hochberg adjusted $p$-values $>0.05$ ).

\section{Agave transposable elements are transcriptionally active} Most plant transposable elements (TEs) are transcriptionally silent [45], they constitute a significant proportion of Agave genomes [28] and contribute to the creation of genetic diversity in many plants [46] including Agave [47,48]. We identified TE-like sequences in agaves (Methods) (Additional file 1: Table S8), the majority of which are derived from retrotransposons (Additional file 1: Table S8). Very few TE annotations encompass entire contigs (Additional file 1: Figure S6), with only 332 contigs in A. tequilana and 171 in $A$. deserti entirely covered by a TE annotation ( $\pm 10 \mathrm{nt}$ from each end). Nearly half of all TE annotations (46.6\%) encompass only the $5^{\prime}$ or $3^{\prime}$ end of transcript contigs (Additional file 1: Figure S7), suggesting that transcription initiation or termination can occur within TEs.

\section{Transcriptome profiles of Agave tissues are distinguished by physiological function}

To examine tissue-specific differences in transcriptome profiles, we analyzed the data sets used for de novo transcriptome assembly based on their tissue of origin (Additional file 1: Table S1). As expected, transcriptome profiles differ between Agave tissues in proportion to their respective physiological functions (Additional file 1: Tables S9 and S10, Figures S8 and S9). For example, very small transcriptome differences are observed between adjacent sections of the $A$. deserti leaf $(r=0.98)$, while the largest differences are observed between roots and leaves in A. tequilana $(r=0.42)$ (Additional file 1: Table S9) and between the distal tip of $A$. deserti leaves and roots $(r=0.39)$ (Additional file 1: Table S10).

In $A$. deserti, we observed consistent higher expression of 13,961 transcripts in samples derived from folded leaves and meristematic tissues (Methods) (Additional file 1: Figure S9). GO-terms enriched within these transcripts include functions related to DNA synthesis, lipid and membrane synthesis, and targeting of proteins to cellular membranes (Additional file 1: Table S11), all activities typically enriched in actively growing cells within developing leaves and meristems. Two enriched GO terms (DNA integration (GO:0015074) and RNA-dependent DNA replication (GO:0006278)) relate to TE biological functions, and 1524 of the 13,961 transcripts (11\%) are TE-like sequences. Further analysis confirms TE-like sequences generally exhibit highest levels of expression in $A$. deserti folded leaf and meristem tissues (Additional file 1: Figure S10), consistent with developmental relaxation of transposable element silencing (DRTS), observed in meristematic tissues of other monocotyledonous plants [49].

\section{Transcriptomic insights into the $A$. deserti proximal-distal leaf axis}

Monocotyledonous leaves develop along a proximal-distal (PD) gradient, maturing from the distal end (leaf tip) to the proximal end (base) [50], and therefore offer an opportunity to assess developmentally regulated gene expression [51]. As leaves are involved in bioenergy-relevant traits (e.g. photosynthesis) and directly face environmental stresses, we sought to gain general insights into the biology of $A$. deserti leaves (Figure 4A). A. deserti loci were divided into 6 major clusters based on expression patterns across four PD sections of the leaf (Figure 4A, 4B) (Methods). GO enrichment analyses identifies biological functions enriched in each cluster (Additional file 1: Tables S12-S17). Clusters A and $\mathrm{B}$, including genes with highest expression at the leaf base, include many loci encoding regulatory proteins, as well as proteins involved in cell wall biogenesis, cellulose synthesis, and carbohydrate synthesis. Clusters E and F, containing genes expressed highest in distal portions of the leaf, include GO terms related to photosynthesis, chlorophyll biosynthesis, and additional regulatory proteins. Taken together, clustering data support the notion that growth and organizational processes occur in basal portions of the leaf while many important energy-related metabolic processes occur at the distal end. General patterns of gene expression for select biological functions were visualized along the leaf PD axis (Figure 4C and Additional file 2). Genes involved in photosynthesis are universally expressed higher in the distal portion of the leaf (Figure 4C), including genes involved in the diurnal shuttling of $\mathrm{CO}_{2}$ in $\mathrm{CAM}$ plants (Additional file 1: Table S18). This suggests that medial-distal portions of $A$. deserti juvenile leaves are the major site of photosynthesis. On the other hand, the basal portion of the leaf is the site of many developmental processes, including cell wall, lignin, and cellulose biosynthesis, stomata development and patterning, and epicuticular wax and suberin biosynthesis (Figure 4C).

We also examined expression of several classes of developmentally-important plant transcription factors and hormones (Figure 4D). Most transcription factor families are expressed highest at the leaf base. Notable exceptions to this pattern are the Class II KNOX genes, which tend to have broad patterns of expression [52], 


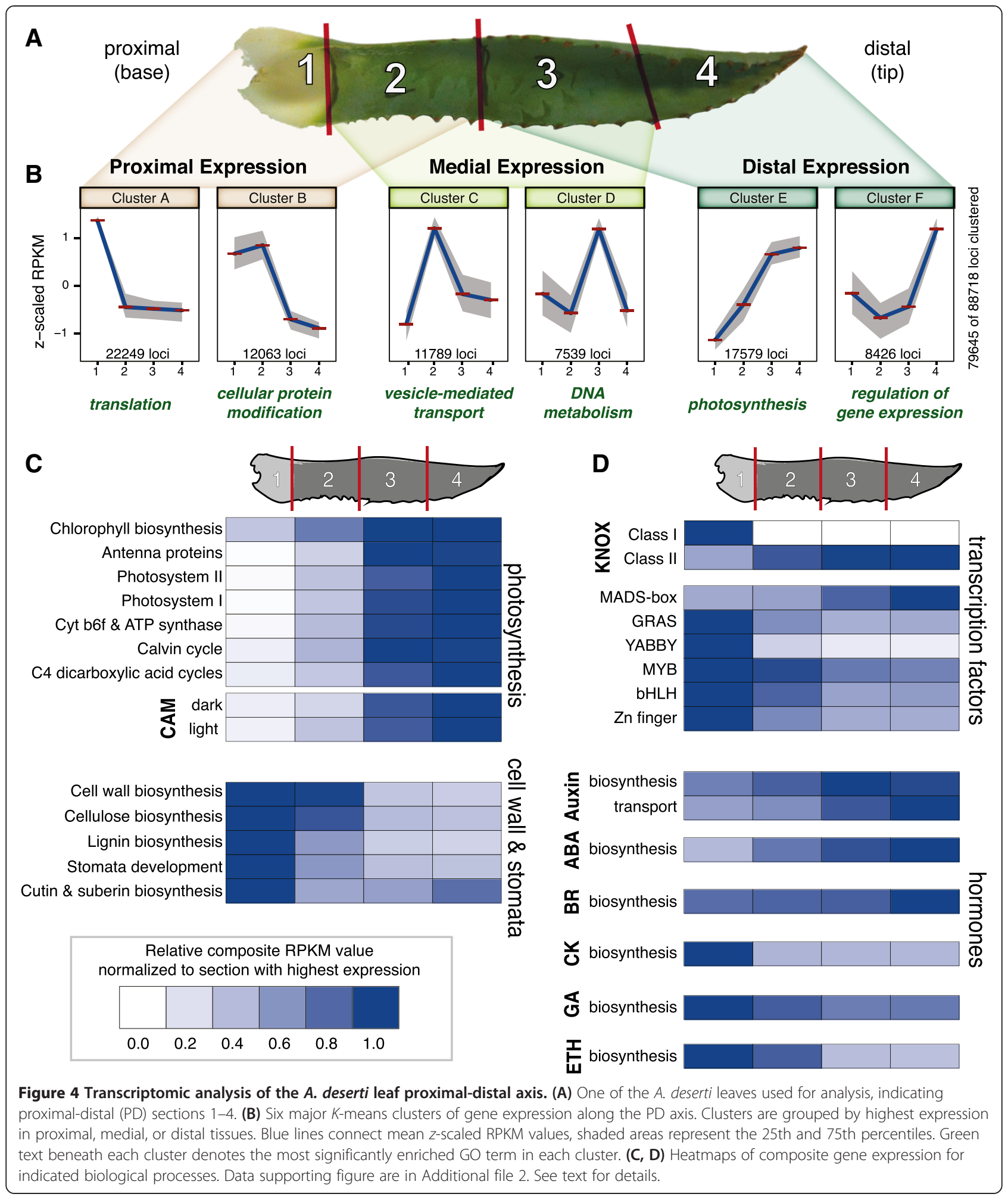

and MADS-box transcription factors, which regulate diverse developmental processes [53]. Hormone synthesis genes are also expressed in gradients along the leaf PD axis (Figure 4D) consistent with their roles in leaves
[54,55]. We observed general PD patterns for auxin, abscisic acid (ABA), brassinosteroids (BR), cytokinin $(\mathrm{CK})$, gibberellin $(\mathrm{GA})$, and ethylene (ETH) hormone biosynthesis (Figure 4D). Taken together, the general 
patterns observed along the PD axis of the $A$. deserti leaf mirror those seen in the monocotyledonous grass Zea mays [51], with transcription factors regulating developmental processes expressed mostly at the leaf base, and functions of the mature leaf, such as photosynthesis, occur more toward the distal end.

\section{Discussion and conclusion}

The transcriptomes of two agaves adapted to semi-arid (A. tequilana) and xeric ( $A$. deserti) environments offer new resources in which to study CAM photosynthesis and other physiological adaptations to prolonged drought and heat. Comparisons of the Agave de novo transcriptome assemblies to other agave sequences and cross-species proteomic comparisons suggest the de novo assemblies are largely complete and accurate. However, the transcriptomes alone provide limited insight into how agaves survive in their environments. For example, though we have identified known genes central to CAM biochemistry in Agave (Additional file 1: Table S18), a full understanding of CAM biology in Agave requires studying the regulation of photosynthetic genes in response to physiological and environmental conditions [25,56,57]. This highlights the need to further functional understanding of Agave transcriptomes through experimentation. Our reference transcriptomes enable molecular investigations of agaves under environmentally controlled conditions to further elaborate the coordinated gene expression underlying CAM, drought resistance, and heat tolerance. As agaves are distinguished from other monocotyledonous plants by regulatory diversity (Additional file 1: Table S7), agave responses to stress may differ from other plants in novel ways.

A simple hypothesis is that agaves adapted to their environments by the expansion of gene families, and the Agave transcriptomes allow preliminary analyses of gene duplication. However, our analysis of inferred Agave proteomes and those of 11 other plant species in the PTS found no solid evidence of gene family expansion in agaves. We cannot, however, rule out the possibility of undetected gene family expansion for two reasons. Firstly, OrthoMCL, our clustering algorithm of choice, is relatively strict compared to alternative clustering algorithms [58], potentially leading to false negative results. Secondly, as agaves are paleopolyploids [27] and gene duplication events cannot be resolved cleanly without a reference genome, expansion of gene families with highly similar sequences will go undetected. More detailed studies of the extent and nature of Agave gene duplications will need to be addressed with a completed genome sequence.

Analyses of the inferred Agave proteomes by Thermorank also failed to find solid evidence of large-scale protein adaptations to thermal stress. In fact, Agave proteomes appear to be no more or less thermostable than those of other land plants (Additional file 1: Figure S5B). Interestingly, the proteome of the green algae Chlamydomonas reinhardtii showed the lowest overall thermostability of the 11 species within Phytozome Tester Set (Additional file 1: Figure S5B)-an expected result given its aquatic habitat-suggesting Thermorank can detect broad differences in protein thermostability. However, to detect more subtle differences between land plant proteomes, more robust methods using protein structure and molecular dynamic simulations [59] may be needed to resolve more subtle protein adaptations to thermal stress.

The de novo transcriptome assemblies are useful to develop molecular markers for further efforts in agave breeding or molecular studies, and we used our data to generate tables of polymorphic sites in the standardized VCF format [60]. Given the species of Agave studied here are primarily outcrossing [61,62] and more often reproduce clonally [5], we expected to find a large number of polymorphisms within the transcriptome. Consistent with this hypothesis, large percentages of both the $A$. deserti and $A$. tequilana loci display SNPs and indels. We also observed a significantly higher frequency of polymorphisms in A. tequilana than $A$. deserti, consistent with the source of the materials, as the $A$. deserti sequence data was generated from two sibling plants, while $A$. tequilana sequence data was generated from a population of individuals (Additional file 1: Table S1). The true number of heterozygous loci may be much higher as alleles not exhibiting equal expression may escape detection with our RNA-seq analyses.

Our analysis of gene expression along the proximal-distal axis of the juvenile $A$. deserti leaf demonstrates core classes of genes and biological processes are similar to those observed in Zea mays [51], supporting evolutionary conservation of monocotyledonous leaf development models [50]. A contrast of $A$. deserti to Zea mays is the expression pattern of MADS-box transcription factors. MADS-box genes can vary widely in expression and function in Agave floral structures and meristems [63], but their role in leaf developmental or metabolic processes remains unknown. The location of auxin biosynthesis occurs at a comparatively more distal portion of the blade in $A$. deserti than $Z$. mays [51]. These distinctions between Agave and Zea mays could be related to morphological differences between the two species: unlike maize leaves, $A$. deserti leaves are lanceolate-shaped with marginal spines [5] and have distinct parenchyma (water storing) and chlorenchyma (photosynthetic) tissues characteristic of succulent plants [19]. Such differences in key developmental transcription factors and hormones are perhaps not unexpected as these may be major determinants of Agave morphological adaptations to xeric environments. 
Our Agave transcriptomes exemplify the power of de novo transcriptome assembly from short-read RNA-seq data [31], which provides both a high-quality sequence resource and insights through transcriptome profiling. Leveraging annotation tools and the scientific work from model plant species facilitated insights into the biology Agave. This rapid production of comprehensive sequence resources for additional species of industrial and biotechnological interest is needed to meet challenges of climate change and bioenergy development [64]. Our de novo Agave transcriptome assemblies provide a guide for such future de novo transcriptome assembly projects. Additional improvements in sequencing length, accuracy, cost, and throughput will make de novo transcriptome assembly an increasingly attractive option for rapid transcriptome exploration.

\section{Methods}

\section{Plant materials}

A. tequilana plants were collected from an A. tequilana plantation in Guanajuato, Mexico. Leaf, root, and stem tissue was collected from 2 different adult plants, each approximately 4 years of age. Juvenile plants from the same field, each approximately 1 year old, were dissected. Equal weights of juvenile roots, leaves, and stems were pooled prior to RNA preparation. A. deserti juveniles were obtained from a local commercial provider (Berkeley, CA) and verified using morphological keys [5]. Plants and tissues were dissected as described (Additional file 1: Table S1, Figure S1). A. deserti tissues were collected from well-watered plants near mid-day.

\section{Molecular methods}

Agave tequilana RNA was extracted from tissues as described previously [65]. A. deserti RNA was prepared with modifications as follows. Tissues were finely sliced and immediately frozen in liquid nitrogen. Approximately $3 \mathrm{~g}$ of plant tissue were finely ground using liquid nitrogen in a mortar and pestle. $7.5 \mathrm{ml}$ Trizol (Invitrogen, Carlsbad, CA) and $1.5 \mathrm{ml}$ chloroform was added and tissue was homogenized. Homegenate was incubated for $10 \mathrm{~min}$ at room temperature and centrifuged $4000 \mathrm{~g}$. Aqueous phase was removed, mixed with 1 volume of a 1:1 phenol:chloroform, and centrifuged at $4000 \mathrm{~g}$. Resulting aqueous phase was mixed with an equal volume of isopropanol and $1 / 10$ volume $5 \mathrm{M} \mathrm{NaCl}$. RNA was precipitated at $-20^{\circ} \mathrm{C}$ overnight, then centrifuged at 10,000 g. RNA pellet was suspended in $500 \mu \mathrm{l}$ RNAsefree $\mathrm{H}_{2} \mathrm{O}$. Phenol:Chloroform extraction was repeated as above. Aqueous phase was mixed with 0.6 volumes of $7 \mathrm{M}$ $\mathrm{LiCl}$ and incubated at $-20^{\circ} \mathrm{C}$ for 40 minutes prior to centrifugation at full speed in a table top microcentrifuge. RNA pellet was rinsed in $70 \%$ ethanol, air-dried briefly, and suspended in $250 \mu \mathrm{l} \mathrm{H} \mathrm{H}_{2} \mathrm{O}$.
Illumina short-read library construction and sequencing Each library construction was initiated with $25 \mu \mathrm{g}$ total RNA. Polyadenylated RNA was selected using the $\mu$ MACS mRNA isolation kit (Miltenyi Biotec, Auburn, CA) and repeated as necessary until rRNA constituted less than $5 \%$ of the remaining purified mRNA before hydrolysis into 250 and 500 nt fragments using RNA Fragmentation Reagents (Ambion, Austin TX). First strand cDNA synthesis was performed using SuperScript II Reverse Transcriptase (Invitrogen, Carlsbad, CA) primed with $3 \mu \mathrm{g}$ random hexamers and $2.5 \mathrm{mM}$ dNTPs. 2nd strand cDNA was prepared in a $100 \mu$ reaction with $2 \mathrm{U}$ RNAseH, 40U DNA Pol I, and 10U DNA ligase with $0.3 \mathrm{mM}$ of each dNTP (with dUTP in place of dTTP). Samples were incubated for 2 hours at $16^{\circ} \mathrm{C}$. Ten units T4 DNA polymerase was added and incubation was continued for an additional 5 minutes. 2nd strand cDNAs were size selected to either 250 or $500 \mathrm{bp}$ using the Pippin Prep system (Sage Science, Beverly, MA). Indexed TruSeq libraries were constructed using manufacturer directions (Illumina, San Diego, CA). dUTP-labeled strands were destroyed using AmpErase uracil $\mathrm{N}$-glycosylase (Applied Biosystems, Foster City, CA). Libraries were amplified through 10 cycles of PCR using Illumina guidelines. Sequencing was performed at the DOE Joint Genome Institute on an Illumina HiSeq 2000 with TruSeq SBS-v3 reagents (Illumina).

\section{Long-read sequencing}

Libraries for Pacific Biosciences single molecule real time (SMRT) sequencing were prepared from A. tequilana 2 nd strand cDNAs (see above). Library were constructed according to manufacturers' guidelines (Pacific Biosciences, Menlo Park, CA) and sequenced on 5 SMRT cells for a total of 751,460 reads. Reads were filtered to remove library artifacts, resulting in 9913 read sequences composed of 27,787 subreads. Filtered Pacific Biosciences subread sequences $\geq 300$ nt were corrected with $114,901,038$ A. tequilana Illumina reads using methods described previously [66]. From this, 4767 successfully corrected, high quality PacBio subreads were returned. To compare the Pacific Biosciences sequencing to the Illumina de novo assembly, corrected PacBio subreads were aligned to the Illumina $A$. tequilana assembly using BLAT [33] with a minimum threshold of $90 \%$ sequence identity.

\section{De novo transcriptome assembly and analyses}

de novo transcriptome assembly of Illumina sequence was performed by Rnnotator [29]. Transcript contigs were binned into loci based on a minimum of 200 bp sequence overlap as determined by an all-vs-all comparison using Vmatch [67]. Following assembly, transcripts were assigned an RPKM [68] value based on the number of uniquely mapping reads aligning to each transcript using 
BWA [69]. Each transcript version per locus was numbered according to its relative abundance for that locus (with version 1 being the most abundant). Transcripts present at less than $10 \%$ of the version 1 transcript were noted as potential precursor transcripts. Transcripts are named by their respective locus, version (isoform) number, raw RPKM and precursor flag; e.g. Locus1v2rpkm3.45_PRE is the 2nd most abundant isoform of Locus 1 with an RPKM of 3.45, marked as a precursor transcript. Additional details about the design and operation of Rnnotator can be found online [70].

\section{Filtering assemblies for high-confidence Agave transcriptomes}

MEGAN v4.621 build 27 [71] was used to identify de novo assembled transcript contigs with homology to plant sequences and filtered RepeatMasker v. open-3.2.9 [72] and DeconSeq v 0.4.1 [73] with a contaminating match equivalent to $>=94 \%$ identity over $90 \%$ of the contig length. Sequences unidentified by MEGAN, Deconseq, or Repeatmasker were either retained or removed from the Agave datasets using their abundance (measured in RPKM) assuming most RNA in the sample originates from agave. Thresholds were defined by the lower quartile RPKM of high-confidence plant contigs. Contigs meeting or exceeding this RPKM were retained within the agave datasets (RPKM $>=0.42$ for $A$. deserti, $\mathrm{RPKM}>=1.2$ for A. tequilana).

\section{Protein prediction, annotation, and clustering}

Open reading frames were annotated using EMBOSS getorf [74] with a maximum length of $1 \times 10^{6}$ and a minimum length of 30 amino acids. Working-set proteomes include only proteins encoded on the + strand of $\mathrm{v} 1$ (most abundant) transcript isoforms, where each protein must be at least 76 aa in length with a CDS encompassing $>=50 \%$ of transcript length. Minimum protein lengths of 76 aa represents the 5 th percentile of protein lengths within the Phytozome Tester Set (below). Pfam, Interpro, and GO annotation was performed using InterProScan [75]. KEGG annotation [76] was retrieved using KAAS [77]. TEs were identified using RepeatMasker (version open-3.2.9) [72] with RepBase Update 2009-06-04 [78].

\section{Phytozome tester set and protein clustering}

The Phytozome Tester Set (PTS) includes select proteomes from Phytozome v8 [35]: Arabidopsis thaliana (TAIR release 10), Brachypodium distachyon (JGI v1.0 8X assembly of $B d 21$ and MIPS/JGI v1.2 annotation), Chlamydomonas reinhardtii (Augustus u10.2 annotation of JGI assembly v4), Glycine max (JGI Glyma1.0 annotation of Glyma1 assembly), Medicago truncatula (Medicago Genome Sequence Consortium release Mt 3.0),
Oryza sativa japonica (MSU Release 7.0), Populus trichocarpa (JGI release v2.0, annotation v2.2), Ricinus communis (TIGR release 0.1), Setaria italica (JGI 8.3X chromosome-scale assembly release 2.0, annotation version 2.1), Sorghum bicolor (Sbi1.4 models from MIPS/PASA on v1.0 assembly), and Zea mays (Maize Genome Project 5b.60 B73). Proteins were binned into orthologous groups (Plant OGs) by OrthoMCL v2.0.3 [34] using default settings.

\section{SNP and indel detection}

All paired-end reads from $A$. deserti libraries $(1,231,372,300$ reads) and randomly selected $A$. tequilana reads (993,931, 796 reads) were used to detect SNPs and indels (polymorphisms). A. deserti reads were aligned to the $A$. deserti v1 transcript contigs and $A$. tequilana reads were aligned to the $A$. tequilana v1 transcript contigs using BWA [69]. Polymorphisms within the v1 transcripts, serving as a proxy for a genomic locus, were called using SAMtools mpileup [60]. Based on the quality value distribution of SNPs and indels (Additional file 1: Figure S4A), only those with a quality score of 999 were considered for further analysis, minimizing low-confidence polymorphism calls from poor sequence quality or low-coverage.

\section{Protein thermostability prediction}

In order to computationally predict thermostability for large protein datasets, we used the core scoring function of Thermorank [44] to assign a thermostability score to each protein as follows:

$$
\begin{aligned}
\text { Thermostability }= & (K \bullet 0.75)+(E \bullet 0.2)+(\text { Pos } \bullet 0.8)+(\text { Chg } \bullet 0.2) \\
& +(\text { Sml } \bullet-0.2)+(\text { Tiny } \bullet-0.2)+(A \bullet-0.3) \\
& +(Q \bullet-0.1)+(T \bullet-0.02)+(\text { ASA } \bullet 0.9)
\end{aligned}
$$

Where $K$ is the molar fraction of lysine, $E$ is the molar fraction of glutamic acid, Pos is the molar fraction of positively charged amino acids ( $\mathrm{R}, \mathrm{H}$ and $\mathrm{K}), \mathrm{Ch} g$ is the molar fraction of charged amino acids (D, E, H, K, and R), $S m l$ is the molar fraction of 'small' amino acids (A, C, D, G, N, P, $\mathrm{S}, \mathrm{T}$, and $\mathrm{V})$, Tiny is the molar fraction of 'tiny' amino acids (A, C, G, S, and T), $T$ is the molar fraction of threonine. $A S A$ is calculated as follows: The residue surface accessible area for each amino acid residue (R) in a hypothetical Gly-R-Gly tripeptide was indexed to data obtained by Chothia [79]. The sum surface area for the peptide is divided by the number of amino acids to obtain an average residue surface area. The average surface area (possible minimum of 75 square angstroms $\left(\AA^{2}\right)$ and maximum of $255 \AA^{2}$ [79]) is divided by $180 \AA^{2}$ (the range between $75 \AA^{2}$ and $255 \AA^{2}$ ) to create a dimensionless value between 0 and 1 . A test of 5000 artificial peptide sequences of random length and 
amino acid composition found high correlation between our thermostability score generator and Thermorank (Pearson $r=0.873$, Additional file 1: Figure S5A).

\section{Protein family size and thermostability analyses}

Detection of Agave OrthMCL-defined PlantOG family memberships, and Thermorank scores were determined by performing a Wilcoxon rank sum text against data obtained from the Phytozome Tester Set. Prior to analysis of PlantOG membership, protein identifiers from agaves and the Phytozome Tester Set were parsed to select non-redundant representative proteomes with a single version 1 (or similarly-labeled) representative protein model per locus. Final $p$-values were corrected for multiple comparisons by the Benjamini-Hochberg procedure [80].

\section{RNA-seq expression analysis and $K$-means clustering}

Contigs containing rRNA-like sequences as determined by BLASTN [81] (E-value $\leq 10)$ against the SILVA v108 database [82] were removed from reference transcriptomes prior to expression analyses. Reads were trimmed to $36 \mathrm{nt}$ and mapped to reference transcriptome using BWA [69]. The number of reads uniquely aligning to each transcript was normalized by the total number of uniquely-aligning reads in the sample, divided by the length of the uniquely mappable portion of each transcript to obtain an RPKM value [68]. Q-values were obtained as described [83]. $Z$-scaled locus RPKM values were grouped by $K$-means clustering, 6 clusters were chosen based on the least within group sum of squares' method [84]. All enrichment analysis was performed using BiNGO [85] with default settings (hypergeometric test with Benjamini-Hochberg $p$-value correction [80]).

\section{Data availability}

Reads are available through the NCBI Sequence Read Archive (SRA), study accessions [GenBank:SRP019885] (A. tequilana) and [GenBank:SRP019506] (A. deserti). Agave transcriptome assembly contigs meeting NCBI requirements are deposited at the Transcriptome Shotgun Assembly (TSA) accessions A. tequilana: [GenBank: GAHU00000000]; A. deserti: [GenBank:GAHT00000000]. Full sequence assemblies, annotations, OrthoMCL clustering, and expression data for both agave datasets as described are available at the Dryad Digital Repository [36].

\section{Additional files}

Additional file 1: Contains supplementary tables and figures referenced in the main text.

Additional file 2: Data supporting Figure 4.

\section{Abbreviations}

Bp: Base pair; BWA: Burrows-Wheeler Aligner; CAM: Crassulacean acid metabolism; DRTS: Developmental relaxation of transposable element silencing; Gbp: Gigabasepair(s); GO: Gene ontology; nt: nucleotide(s); RBH: Reciprocal best hit; RPKM: Reads per kilobase of exon model per million mapped reads; TE: Transposable element.

\section{Competing interests}

S.G. has received travel reimbursement funds from Illumina Inc. (San Diego, CA). The authors declared that they have no competing interest.

\section{Authors' contributions}

SG, JM, ZW, and AV designed research. SG and JM performed research. JS and MA-J contributed biological materials. SG and AV wrote the manuscript. All authors read and approved the final manuscript.

\section{Acknowledgements}

The authors would like to thank Gerald Tuskan, Xiaohan Yang, Joel Martin, Wendy Schackwitz, Erika Lindquist, Feng Chen, Chia-Lin Wei, Cindy Choi, Natasha Zvenigorodsky, Dongwan Kang, Crystal Wright, Devin Coleman-Derr, Stephen Fairclough, Nicole Johnson, and Gretchen North for technical assistance and comments; and Michael McKain for data access [27]. Funding was provided by Lawrence Berkeley National Laboratory Directed Research and Development Program (LB11036). The work conducted by the U.S. Department of Energy Joint Genome Institute is supported by the Office of Science of the U.S. Department of Energy under Contract No.

\section{DE-AC02-05CH11231.}

\section{Author details}

${ }^{1}$ DOE Joint Genome Institute, Walnut Creek, CA, USA. ${ }^{2}$ Genomics Division, Lawrence Berkeley National Laboratory, One Cyclotron Road, Berkeley, CA 94720, USA. ${ }^{3}$ Department of Genetic Engineering, CINVESTAV, Irapuato, Guanajuato, Mexico.

Received: 25 April 2013 Accepted: 13 August 2013

Published: 19 August 2013

\section{References}

1. Borland AM, Griffiths H, Hartwell J, Smith JAC: Exploiting the potential of plants with crassulacean acid metabolism for bioenergy production on marginal lands. J Exp Bot 2009, 60(10):2879-2896.

2. Nobel PS: Achievable productivities of vertain CAM plants - basis for high values compared with C3 and C4 plants. New Phytol 1991, 119(2):183-205.

3. Woodhouse RM, Williams JG, Nobel PS: Simulation of plant temperature and water loss by the desert succulent, Agave deserti. Oecologia 1983, 57(3):291-297.

4. Nobel PS: Water relations and photosynthesis of a desert CAM plant, Agave deserti. Plant Physiol 1976, 58(4):576-582.

5. Gentry HS: Agaves of continental North America. Tucson, Ariz: University of Arizona Press; 1982.

6. Gates DM, Keegan HJ, Schleter JC, Weidner VR: Spectral properties of plants. Appl Optics 1965, 4(1):11

7. Boom A, Damste JSS, de Leeuw JW: Cutan, a common aliphatic biopolymer in cuticles of drought-adapted plants. Org Geochem 2005, 36(4):595-601

8. Wattendorff J, Holloway PJ: Studies on the ultrastructure and histochemistry of plant cuticles - the cuticular membrane of Agave americana L. in situ. Ann Bot-London 1980, 46(1):13.

9. North GB, Nobel PS: Root-soil contact for the desert succulent Agave deserti in wet and drying soil. New Phytol 1997, 135(1):21-29.

10. North GB, Brinton EK, Garrett TY: Contractile roots in succulent monocots: convergence, divergence and adaptation to limited rainfall. Plant Cell Environ 2008, 31(8):1179-1189.

11. Nobel PS: Desert wisdom/agaves and cacti : $\mathrm{CO} 2$, water, climate change. New York: iUniverse; 2010.

12. Garcia-Moya E, Romero-Manzanares A, Nobel PS: Highlights for Agave productivity. Gcb Bioenergy 2011, 3(1):4-14.

13. Somerville C, Youngs H, Taylor C, Davis SC, Long SP: Feedstocks for lignocellulosic biofuels. Science 2010, 329(5993):790-792. 
14. Davis AS, Dohleman F, Long SP: The global potential for Agave as a biofuel feedstock. Gcb Bioenergy 2011, 3(1):68-78.

15. Cedeno M: Tequila production. Crit Rev Biotechnol 1995, 15(1):1-11.

16. Valenzuela-Zapata AG, Nabhan GP: Tequila : a natural and cultural history. Tucson: University of Arizona Press; 2003

17. Distilled Spirits Council of the United States: U.S. tequila market at a glance. Washington, D.C: Distilled Spirits Council of the United States; 2011.

18. Valenzuela A: A new agenda for blue agave landraces: food, energy and tequila. Gcb Bioenergy 2011, 3(1):15-24.

19. Nobel PS: Environmental biology of agaves and cacti. Cambridge; New York: Cambridge University Press; 1988.

20. Nobel PS, Hartsock TL: Temperature, water, and PAR influences on predicted and measured productivity of Agave deserti at various elevations. Oecologia 1986, 68(2):181-185.

21. Zabriskie JG: Plants of Deep Canyon and the Central Coachella Valley, California. Riverside, CA: Philip L. Boyd Deep Canyon Desert Research Center, Univeristy of California, Riverside; 1979.

22. Jordan PW, Nobel PS: Infrequent establishment of seedlings of Agave deserti (Agavaceae) in the northwestern Sonoran desert. Am J Bot 1979, 66(9):1079-1084

23. Nobel PS, Smith SD: High and low temperature tolerances and their relationships to distribution of agaves. Plant Cell Environ 1983, 6(9):711-719.

24. Nobel PS: Productivity of Agave deserti - measurement by dry-weight and monthly prediction using physiological-responses to environmental parameters. Oecologia 1984, 64(1):1-7.

25. Nobel PS, Valenzuela AG: Environmental responses and productivity of the CAM plant, Agave tequilana. Agr Forest Meteorol 1987, 39(4):319-334.

26. Palomino G, Dolezel J, Mendez I, Rubluo A: Nuclear genome size analysis of Agave tequilana Weber. Caryologia 2003, 56(1):37-46.

27. McKain MR, Wickett N, Zhang Y, Ayyampalayam S, McCombie WR, Chase MW, Pires JC, Depamphilis CW, Leebens-Mack J: Phylogenomic analysis of transcriptome data elucidates co-occurrence of a paleopolyploid event and the origin of bimodal karyotypes in Agavoideae (Asparagaceae). Am J Bot 2012, 99(2):397-406.

28. Bousios A, Saldana-Oyarzabal I, Valenzuela-Zapata AG, Wood C, Pearce SR: Isolation and characterization of Ty1-copia retrotransposon sequences in the blue agave (Agave tequilana Weber var. azul) and their development as SSAP markers for phylogenetic analysis. Plant Sci 2007, 172(2):291-298.

29. Martin J, Bruno VM, Fang Z, Meng X, Blow M, Zhang T, Sherlock G, Snyder M, Wang Z: Rnnotator: an automated de novo transcriptome assembly pipeline from stranded RNA-seq reads. BMC Genomics 2010, 11:663.

30. Zerbino DR, Birney E: Velvet: algorithms for de novo short read assembly using de Bruijn graphs. Genome Res 2008, 18(5):821-829.

31. Martin JA, Wang Z: Next-generation transcriptome assembly. Nat Rev Genet 2011, 12(10):671-682

32. Eid J, Fehr A, Gray J, Luong K, Lyle J, Otto G, Peluso P, Rank D, Baybayan P, Bettman $B$, et al: Real-time DNA sequencing from single polymerase molecules. Science 2009, 323(5910):133-138

33. Kent WJ: BLAT-the BLAST-like alignment tool. Genome Res 2002, 12(4):656-664

34. Fischer S, Brunk BP, Chen F, Gao X, Harb OS, lodice JB, Shanmugam D, Roos DS, Stoeckert CJ Jr: Using OrthoMCL to assign proteins to OrthoMCL-DB groups or to cluster proteomes into new ortholog groups. Curr Protoc Bioinformatics 2011, Chapter 6:Unit 612 11-19.

35. Goodstein DM, Shu S, Howson R, Neupane R, Hayes RD, Fazo J, Mitros T, Dirks W, Hellsten U, Putnam N, et al: Phytozome: a comparative platform for green plant genomics. Nucleic Acids Res 2012, 40(Database issue):D1178-D1186.

36. Dryad Digital Repository. [http://datadryad.org/resource/doi:10.5061/dryad.h5t68]

37. Angiosperm Phylogeny Group: An update of the angiosperm phylogeny group classification for the orders and families of flowering plants: APG III. Bot J Linn Soc 2009, 161:105-121.

38. Janssen $T$, Bremer $K$ : The age of major monocot groups inferred from 800 + rbcL sequences. Bot J Linn Soc 2004, 146(4):385-398.

39. Magallon S, Castillo A: Angiosperm diversification through time. Am J Bot 2009, 96(1):349-365

40. Timperio AM, Egidi MG, Zolla L: Proteomics applied on plant abiotic stresses: role of heat shock proteins (HSP). J Proteomics 2008, 71(4):391-411.

41. Scharf KD, Berberich T, Ebersberger I, Nover L: The plant heat stress transcription factor (Hsf) family: structure, function and evolution. Biochim Biophys Acta 2012, 1819(2):104-119.
42. Hanin M, Brini F, Ebel C, Toda Y, Takeda S, Masmoudi K: Plant dehydrins and stress tolerance: versatile proteins for complex mechanisms. Plant Signal Behav 2011, 6(10):1503-1509.

43. Granick EB: A karyosystematic study of the genus Agave. Am J Bot 1944, 31(5):283-298

44. Li Y, Middaugh CR, Fang J: A novel scoring function for discriminating hyperthermophilic and mesophilic proteins with application to predicting relative thermostability of protein mutants. BMC Bioinforma 2010, 11:62.

45. Lisch D, Bennetzen JL: Transposable element origins of epigenetic gene regulation. Curr Opin Plant Biol 2011, 14(2):156-161.

46. Lisch D: How important are transposons for plant evolution? Nat Rev Genet 2012, 14(1):49-61.

47. Torres-Moran MI, Escoto-Delgadillo M, Molina-Moret S, Rivera-Rodriguez DM, Velasco-Ramirez AP, Infante D, Portillo L: Assessment of genetic fidelity among Agave tequilana plants propagated asexually via rhizomes versus in vitro culture. Plant Cell Tiss Org 2010, 103(3):403-409.

48. Infante D, Molina S, Demey JR, Gamez E: Asexual genetic variability in Agavaceae determined with inverse sequence-tagged repeats and amplification fragment length polymorphism analysis. Plant Mol Biol Rep 2006, 24(2):205-217.

49. Martinez G, Slotkin RK: Developmental relaxation of transposable element silencing in plants: functional or byproduct? Curr Opin Plant Biol 2012, 15:496-502.

50. Freeling M: A conceptual framework for maize leaf development. Dev Biol 1992, 153(1):44-58.

51. Li P, Ponnala L, Gandotra N, Wang L, Si Y, Tausta SL, Kebrom TH, Provart N, Patel $R$, Myers $C R$, et al: The developmental dynamics of the maize leaf transcriptome. Nat Genet 2010, 42(12):1060-1067.

52. Hake S, Smith HM, Holtan H, Magnani E, Mele G, Ramirez J: The role of knox genes in plant development. Annu Rev Cell Dev Biol 2004, 20:125-151.

53. Smaczniak C, Immink RG, Angenent GC, Kaufmann K: Developmental and evolutionary diversity of plant MADS-domain factors: insights from recent studies. Development 2012, 139(17):3081-3098.

54. McSteen P: Auxin and monocot development. Cold Spring Harb Perspect Biol 2010, 2(3):a001479

55. Blein T, Hasson A, Laufs P: Leaf development: what it needs to be complex. Curr Opin Plant Biol 2010, 13(1):75-82.

56. Silvera K, Neubig KM, Whitten WM, Williams NH, Winter K, Cushman JC: Evolution along the crassulacean acid metabolism continuum. Funct Plant Biol 2010, 37(11):995-1010.

57. Hartsock TL, Nobel PS: Watering converts a CAM plant to daytime $\mathrm{CO}_{2}$ uptake. Nature 1976, 262:574-576.

58. Chen F, Mackey AJ, Vermunt JK, Roos DS: Assessing performance of orthology detection strategies applied to eukaryotic genomes. PLOS One 2007, 2(4):e383.

59. Sterpone F, Melchionna S: Thermophilic proteins: insight and perspective from in silico experiments. Chem Soc Rev 2012, 41(5):1665-1676.

60. Li H, Handsaker B, Wysoker A, Fennell T, Ruan J, Homer N, Marth G, Abecasis G, Durbin R, Genome Project Data Processing S: The sequence alignment/map format and SAMtools. Bioinformatics 2009, 25(16):2078-2079.

61. Molina-Freaner F, Equiarte LE: The pollination biology of two paniculate agaves (Agavaceae) from northwestern Mexico: contrasting roles of bats as pollinators. Am J Bot 2003, 90(7):1016-1024

62. Escobar-Guzman RE, Hernandez FZ, Vega KG, Simpson J: Seed production and gametophyte formation in Agave tequilana and Agave americana. Botany 2008, 86(11):1343-1353.

63. Delgado Sandoval Sdel C, Abraham Juarez MJ, Simpson J: Agave tequilana MADS genes show novel expression patterns in meristems, developing bulbils and floral organs. Sex Plant Reprod 2012, 25(1):11-26.

64. Rubin EM: Genomics of cellulosic biofuels. Nature 2008 454(7206):841-845

65. Martinez-Hernandez A, Mena-Espino ME, Herrera-Estrella AH, Martinez-Hernandez P: Construcción de bibliotecas de ADNc y análisis de expresión génica por RT-PCR en agaves. Revista Latinoamericana de Química 2010, 38:21-42.

66. Koren S, Schatz MC, Walenz BP, Martin J, Howard JT, Ganapathy G, Wang Z, Rasko DA, McCombie WR, Jarvis ED, et al: Hybrid error correction and de novo assembly of single-molecule sequencing reads. Nat Biotechnol 2012, 30(7):693-700. 
67. Vmatch. [http://www.vmatch.de].

68. Mortazavi A, Williams BA, McCue K, Schaeffer L, Wold B: Mapping and quantifying mammalian transcriptomes by RNA-Seq. Nat Methods 2008, 5(7):621-628.

69. Li H, Durbin R: Fast and accurate short read alignment with Burrows-Wheeler transform. Bioinformatics 2009, 25(14):1754-1760.

70. Rnnotator on google code. [https://sites.google.com/a/lbl.gov/rnnotator/].

71. Huson DH, Mitra S, Ruscheweyh HJ, Weber N, Schuster SC: Integrative analysis of environmental sequences using MEGAN4. Genome Res 2011, 21(9):1552-1560

72. Smit AFA, Hubley R, Green P: Repeatmasker open-3.0. http://www.repeatmasker. org. 1996-2010.

73. Schmieder R, Edwards R: Fast identification and removal of sequence contamination from genomic and metagenomic datasets. PLoS One 2011, 6(3):e17288.

74. Rice P, Longden I, Bleasby A: EMBOSS: the European molecular biology open software suite. Trends Genet 2000, 16(6):276-277.

75. Zdobnov EM, Apweiler R: InterProScan-an integration platform for the signature-recognition methods in InterPro. Bioinformatics 2001, 17(9):847-848

76. Kanehisa M, Goto S, Sato Y, Furumichi M, Tanabe M: KEGG for integration and interpretation of large-scale molecular data sets. Nucleic Acids Res 2012, 40(Database issue):D109-D114.

77. Moriya Y, Itoh M, Okuda S, Yoshizawa AC, Kanehisa M: KAAS: an automatic genome annotation and pathway reconstruction server. Nucleic Acids Res 2007, 35(Web Server issue):W182-W185.

78. Jurka J, Kapitonov W, Pavlicek A, Klonowski P, Kohany O, Walichiewicz J: Repbase update, a database of eukaryotic repetitive elements. Cytogenet Genome Res 2005, 110(1-4):462-467.

79. Chothia C: Nature of accessible and buried surfaces in proteins. J Mol Biol 1976, 105(1):1-14.

80. Benjamini $Y$, Hochberg $Y$ : Controlling the false discovery rate: a practical and powerful aproach to multiple testing. J R Stat Soc 1995, 57(1):289-300.

81. Altschul SF, Gish W, Miller W, Myers EW, Lipman DJ: Basic local alignment search tool. J Mol Biol 1990, 215(3):403-410.

82. Pruesse E, Quast C, Knittel K, Fuchs BM, Ludwig W, Peplies J, Glockner FO: SILVA: a comprehensive online resource for quality checked and aligned ribosomal RNA sequence data compatible with ARB. Nucleic Acids Res 2007, 35(21):7188-7196.

83. Herbert JM, Stekel D, Sanderson S, Heath VL, Bicknell R: A novel method of differential gene expression analysis using multiple cDNA libraries applied to the identification of tumour endothelial genes. BMC Genomics 2008, 9:153.

84. Everitt BS, Hothorn T: A handbook of statistical analyses using R. 1st edition. Boca Raton, FL: Chapman and Hall; 2006.

85. Maere S, Heymans K, Kuiper M: BiNGO: a Cytoscape plugin to assess overrepresentation of gene ontology categories in biological networks. Bioinformatics 2005, 21(16):3448-3449.

doi:10.1186/1471-2164-14-563

Cite this article as: Gross et al:: De novo transcriptome assembly of drought tolerant CAM plants, Agave deserti and Agave tequilana. BMC Genomics 2013 14:563.

\section{Submit your next manuscript to BioMed Central and take full advantage of:}

- Convenient online submission

- Thorough peer review

- No space constraints or color figure charges

- Immediate publication on acceptance

- Inclusion in PubMed, CAS, Scopus and Google Scholar

- Research which is freely available for redistribution

Submit your manuscript at www.biomedcentral.com/submit 